\title{
The Effect of Cooling Rate on Microstructure and Mechanical Properties of Zr-Based Bulk Metallic Glasses
}

\author{
Weihong Fu, ${ }^{1}$ Yajuan Sun, ${ }^{1}$ and Wei Zhang ${ }^{2}$ \\ ${ }^{1}$ School of Science, Tianjin Polytechnic University, Tianjin 300387, China \\ ${ }^{2}$ School of Materials Science and Engineering, Hebei University of Technology, Tianjin 300401, China \\ Correspondence should be addressed to Yajuan Sun; sunyajuan1998@126.com
}

Received 4 September 2013; Accepted 28 November 2013

Academic Editor: You Song

Copyright (c) 2013 Weihong Fu et al. This is an open access article distributed under the Creative Commons Attribution License, which permits unrestricted use, distribution, and reproduction in any medium, provided the original work is properly cited.

\begin{abstract}
The aim of the present study is to shed some insights on the effect of cooling rate on the microstructure and mechanical properties for glass-forming alloys. A crystalline gradient was observed in the microstructure of $12 \mathrm{~mm}$ diameter $\mathrm{Zr}_{51} \mathrm{Al}_{9.96} \mathrm{Ni}_{14.34} \mathrm{Cu}_{24.9}(\mathrm{Zr} 51)$ alloy sample from the edge to center due to uneven cooling rates. Microhardness results indicate that the lower the cooling rate, the higher the hardness for the studied alloy.
\end{abstract}

\section{Introduction}

As a novel material, bulk metallic glass (BMG) has developed for several decades and dozens of amorphous alloy systems have been involved in, for example, BMGs based on Mg, La, $\mathrm{Pd}, \mathrm{Zr}, \mathrm{Ti}, \mathrm{Cu}, \mathrm{Ni}, \mathrm{Al}, \mathrm{Fe}, \mathrm{Co}, \mathrm{Nd}, \mathrm{Ce}, \mathrm{Y}$, and so on [1-5]. Due to its disordered atomic structure, metallic glass owns lots of superior physical, chemical, and mechanical properties, such as high elastic limits, high failure strengths and hardness, good toughness and high wear resistance, excellent magnetic properties, and high corrosion resistance $[1,2,6,7]$, which therefore promise it the excellent candidate for applications in modern industry. However, the poor glass forming ability (GFA) prohibits this pace.

As known to many scientists, cooling rate plays an important role in the fabrication of BMGs, although the dependence on it is not as great as the earlier days. According to the three famous empirical rules proposed by Inoue [8], the GFA of metallic glasses could be improved by choosing appropriate compositions. However, experiments indicate that many physical and mechanical properties are very sensitive to the cooling rate during fabrication [9-14]. Obviously, the cooling rate restricts the atomic rearrangement during the glass formation process and affects the properties of BMGs further. Simply speaking, a slow cooling rate will leave more time for this rearrangement, which might even introduce some crystallites into the sample.

The effect of cooling rate on densities has been investigated in $\mathrm{Pb}$-based metallic glasses [9]. It indicates that when the cooling rate decreased from $500 \mathrm{~K} / \mathrm{s}$ to $1.98 \mathrm{~K} / \mathrm{s}$, densities increased from $9.270 \mathrm{~g} / \mathrm{cm}^{3}$ to $9.285 \mathrm{~g} / \mathrm{cm}^{3}$, accompanying an increase in the hardness from $525 \mathrm{Hv}$ to $537 \mathrm{Hv}$. Chen et al. [11] also carried out similar investigations on $\mathrm{Pb}$ Si binary alloy; it is shown that hardness increases with decreasing the cooling rate. Similarly, Yan et al. [13] studied this effect on the microstructure and mechanical properties in Zr-based BMG by using wedge samples. However, systematic investigations in this aspect are still limited. In this paper, we choose the $\mathrm{Zr}-\mathrm{Al}-\mathrm{Ni}-\mathrm{Cu}$ alloy system. And a $12 \mathrm{~mm}$ diameter $\mathrm{Zr}_{51} \mathrm{Al}_{9.96} \mathrm{Ni}_{14.34} \mathrm{Cu}_{24.9}$ (designated as $\mathrm{Zr} 51$ in abbreviation) partially crystallized rod sample was fabricated. Then corresponding to different cooling rate, the evolution of microstructure, thermal, and mechanical properties of different locations from edge to center in the cross-section is studied.

\section{Experimental Procedures}

The $\mathrm{Zr}-\mathrm{Al}-\mathrm{Ni}-\mathrm{Cu}$ alloy ingot was produced by arc melting pure elemental metals of $\mathrm{Zr}$ ( $>99.5 \mathrm{wt} . \%), \mathrm{Al}$ (>99.9 wt.\%), 
$\mathrm{Ni}$ (>99.9 wt.\%), and $\mathrm{Cu}$ (>99.9 wt.\%) together under a highly purified argon atmosphere. Each ingot was remelted for four times to ensure the homogeneous composition. Then $12 \mathrm{~mm}$ diameter rod was fabricated by remelting the button ingots and suction casting the molten alloy into a copper mold. Glassy structure of the as-cast sample was detected by a conventional X-ray diffraction (XRD, Rigaku, D/MaxIIIA) with $\mathrm{Cu}-\mathrm{K}_{\alpha}$ radiation. Thermal properties of the BMG were examined using differential scanning calorimetry (DSC, Perkin-Elmer DSC-7) at a heating rate of $20 \mathrm{~K} / \mathrm{min}$ under a constant flow of argon. Transmission electron microscopy (TEM) observation was carried out on a Philips CM200 operating at $200 \mathrm{kV}$. TEM samples were finally thinned by twin-jet polishing in the bath of liquid nitrogen. Vickers hardness measurements were conducted with a load of $500 \mathrm{~g}$ and holding time of $20 \mathrm{~s}$. Each Vickers hardness value was the average of eight individual measurements. Indentation morphologies were checked by scanning electron microscopy (SEM, Hitachi, S-570).

\section{Results and Discussion}

3.1. Microstructure Evolution with Different Cooling Rates. As we all know, in the rod sample, the real cooling rate decreases from edge to center. Therefore, in this part we will discuss the microstructure evolution from edge to center. The XRD pattern for the $12 \mathrm{~mm}$ diameter $\mathrm{Zr}_{51} \mathrm{Al}_{9.96} \mathrm{Ni}_{14.34} \mathrm{Cu}_{24.9}$ (Zr51) alloy is shown in Figure 1(a). It can be seen that, on the base of the diffuse halo, there exist some sharp peaks which indicate the crystalline phases precipitating during the solidification. In order to examine the detailed nature of the microstructure, cross-section of the sample was investigated. As shown in Figure 1(b), six locations were assigned along the diameter direction. Clearly, they located symmetrically to the center of the circle and were denoted as \#1 \#6. Due to the symmetrically assignment of the six locations, we only choose locations \#1, \#2, and \#3 to study the thermal properties and microstructures.

Thermal analysis results; DSC curves, for locations \#1, \#2, and $\# 3$; and $40 \mu \mathrm{m}$ ribbon samples are shown in Figure 2(a). Clearly, comparing with the ribbon sample, the DSC curve corresponding to the edge part in location \#1 indicates conspicuous glass transition and exothermic crystallization peak which proves the amorphous nature of this part around the edge of the sample. In the medium region indicated by location \#2, the heat of crystallization decreases, although there is still a detectable glass transition. In the center of the sample, no glass transition is observed and the heat of crystallization also decreases largely. According to the method proposed in [14], the volume fraction of crystalline is evaluated as

$$
V_{f}=\frac{\left(\Delta H_{x m}-\Delta H_{x c}\right)}{\Delta H_{x m}} \times 100 \%,
$$

where $V_{f}$ is the volume fraction of crystalline, $\Delta H_{x m}$ is the heat of crystallization when it is full amorphous, and $\Delta H_{x c}$ is the heat of crystallization when there are some crystalline phases in the amorphous sample. Considering

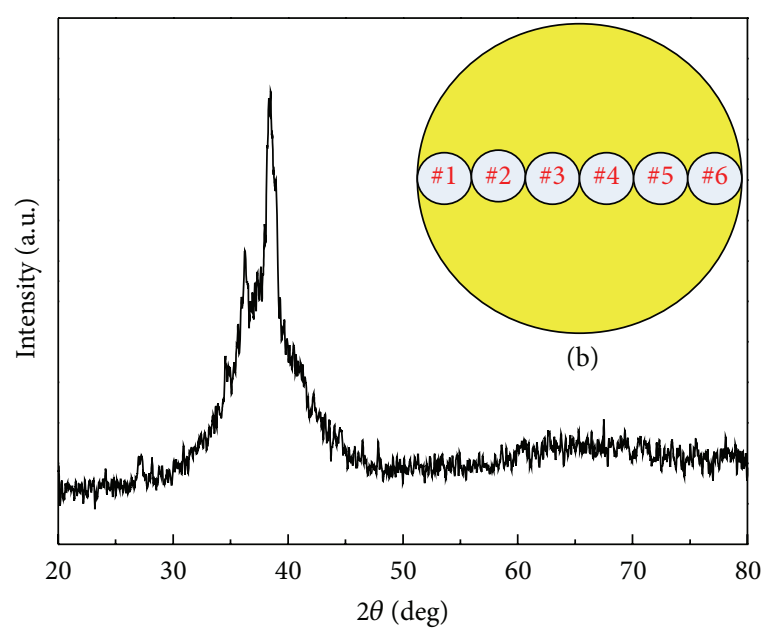

(a)

FIgURE 1: (a) XRD pattern of the as-cast Zr51 alloy with $12 \mathrm{~mm}$ diameter, and (b) diagram of the alloy showing \#1 \#6 locations.

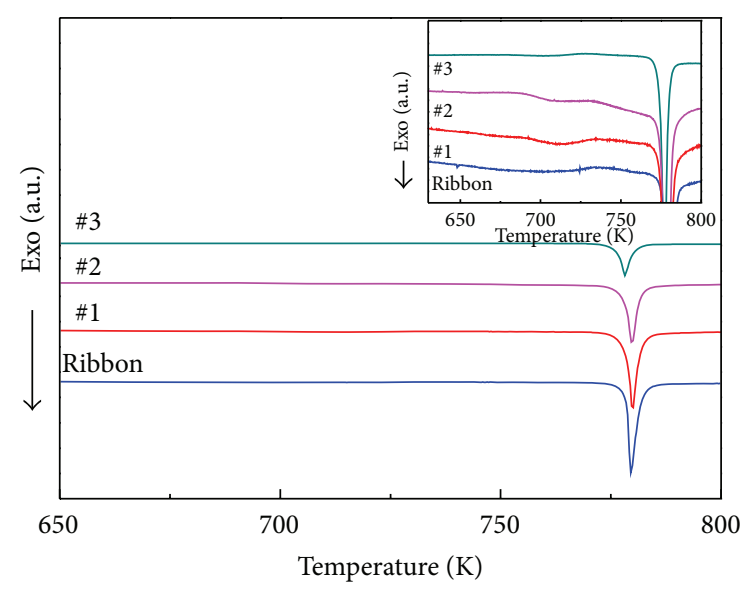

(a)

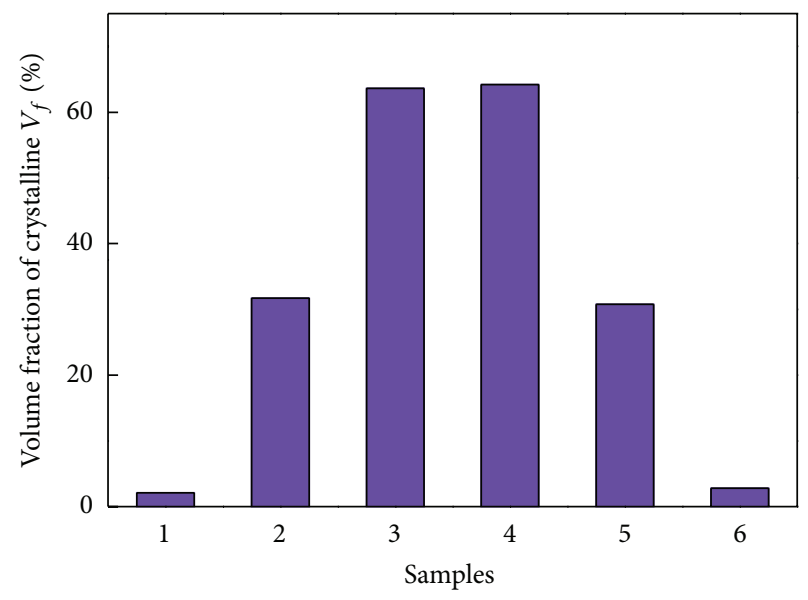

(b)

FIGURE 2: (a) DSC curves of Zr51 alloy for locations \#1, \#2, and \# 3 as well as ribbon sample, (inset in a) an enlarged section below the glass transition temperature, and (b) the volume fraction of crystalline for different locations. 


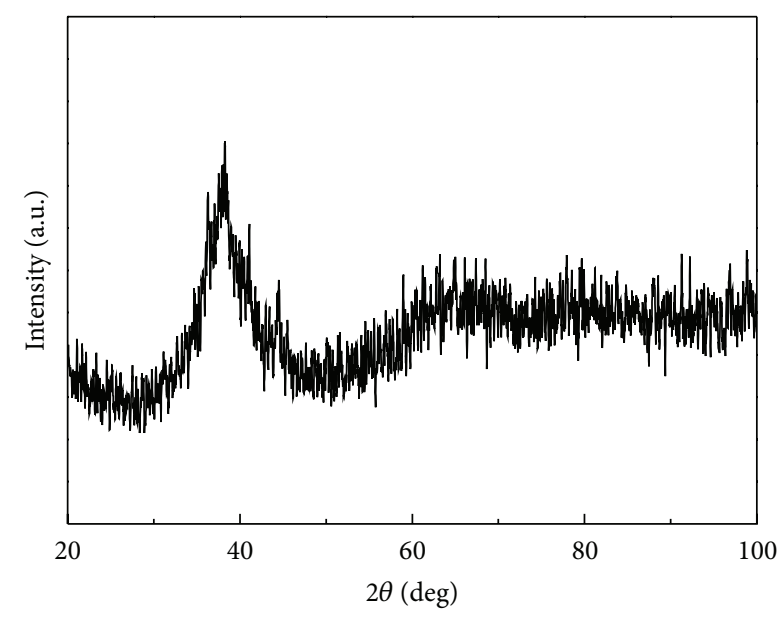

(a)

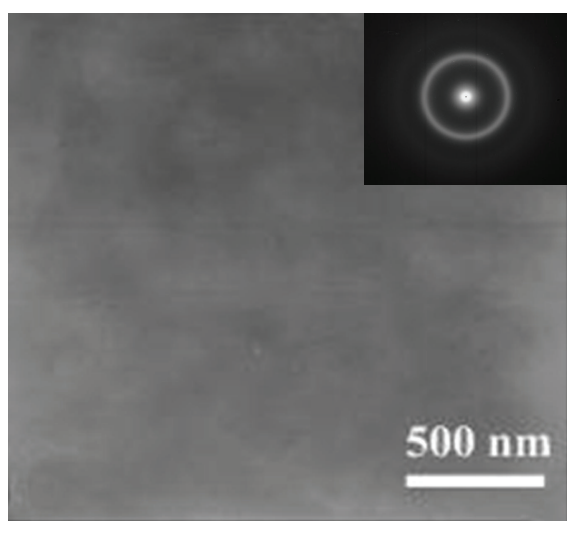

(b)

FIGURE 3: XRD pattern (a) and bright-field TEM image with corresponding SAED pattern (b) for location \#1.

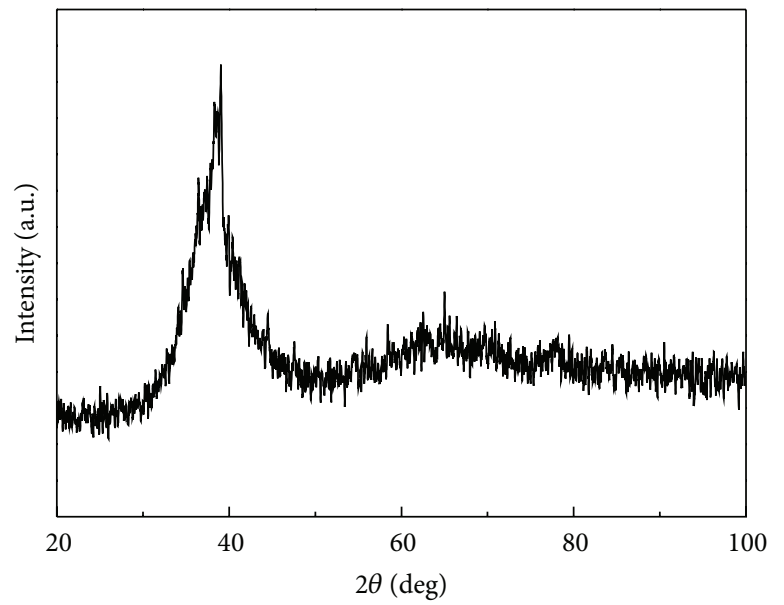

(a)

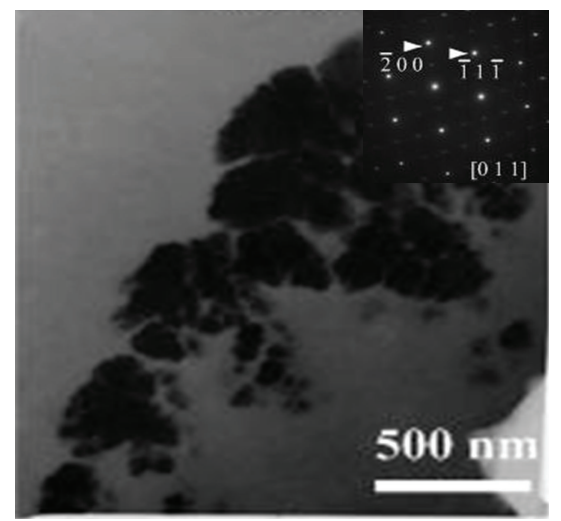

(b)

FIGURE 4: XRD pattern (a) and bright-field TEM image with corresponding SAED patter (b) for location \#2.

the full amorphous nature of the ribbon sample, the volume fraction of the crystalline phases corresponding to locations $\# 1, \# 2$, and \#3 are calculated and displayed in Figure 2(b). As can be seen, the volume fraction of the crystalline phases in the center is higher than that in the edge. This is the result of the decrease of cooling rate from edge to center which then leads to an increasing volume fraction of the crystalline phases.

Analyzing the microstructure of location \#1, a nearly full amorphous matrix is detected. A broad diffraction halo in the XRD pattern indicates the formation of amorphous phase in the as-cast sample (as shown in Figure 3(a)). Furthermore, TEM bright-field image with the corresponding selected area electron diffraction (SAED) in Figure 3(b) indicates that except for diffraction halos, no sharp diffraction ring or spots can be observed. It further confirms that the as-cast sample possesses only glass structure.
Figure 4 displays the microstructure of the medium transition part location \#2 in the cross-section. As shown in Figure 4(a), some weak peaks exist on the base of the diffuse halo, which implies that in this part, with the cooling rate decreasing, certain in situ crystallization happens in the Zr51 alloy. TEM bright-field image in Figure 4(b) indicates that the microstructure of this part is composed of an amorphous matrix and some dispersed crystalline phases, which is consistent with the XRD result. Furthermore, we analyzed the electron diffraction pattern of this crystalline phase. It's found that all of these primary crystallized phases are in the same structure. Figure 4(b) gives out a bright-field image and the electron diffraction pattern of this crystalline phase. Further analysis demonstrates that this crystalline phase is a hexagonal $\mathrm{Zr}_{5} \mathrm{Al}_{4}$ phase (with the lattice parameter of $a=0.8437 \mathrm{~nm}$ and $c=0.58 \mathrm{~nm}$ ). Therefore, it can be concluded that, when the decreased cooling rate cannot 


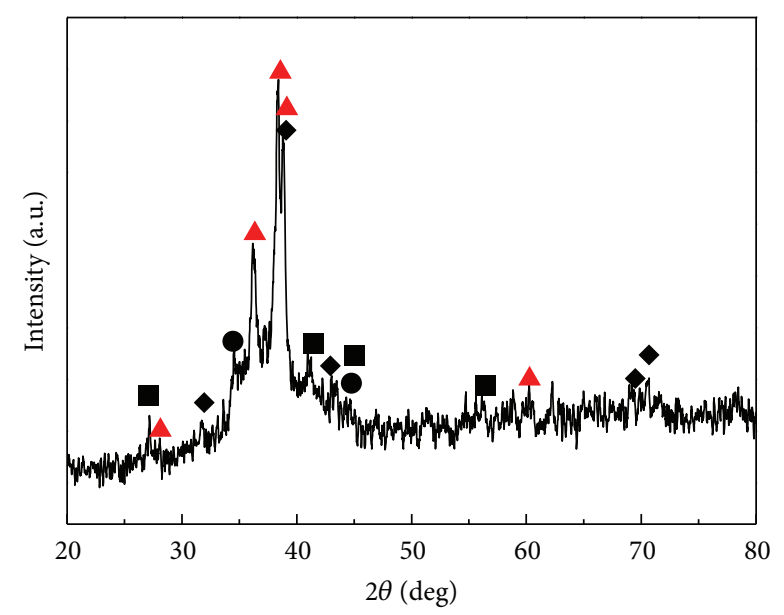
$\mathrm{Al}_{3} \mathrm{Zr}_{5}$
$\rightarrow \mathrm{AlZr}_{3}$
- $\mathrm{Al}_{3} \mathrm{Zr}_{2}$
$\mathrm{Ni}_{10} \mathrm{Zr}_{7}$

(a)

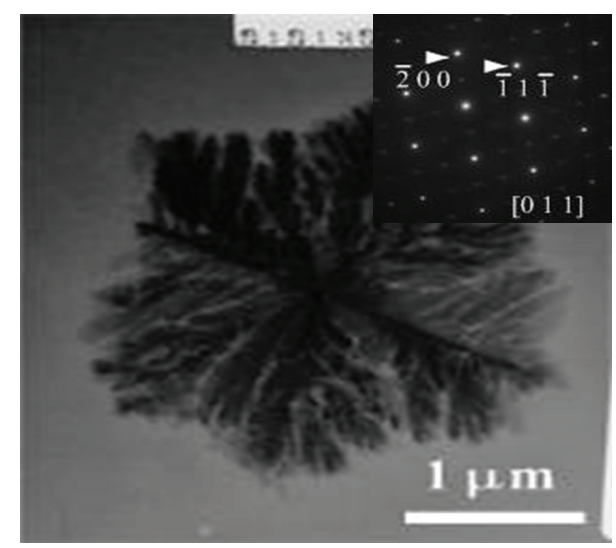

(b)

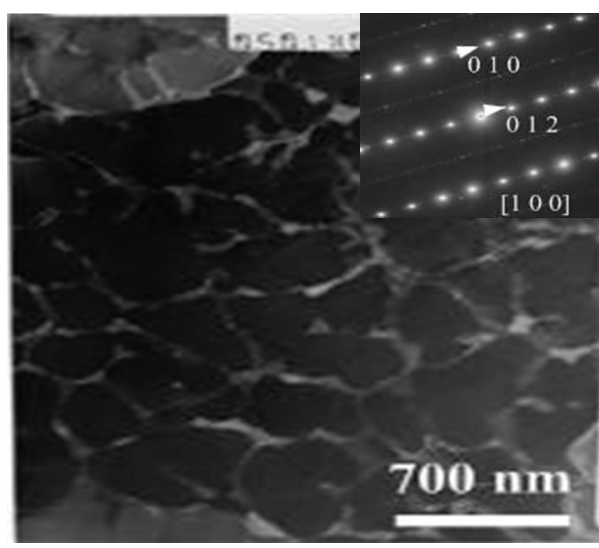

(d)

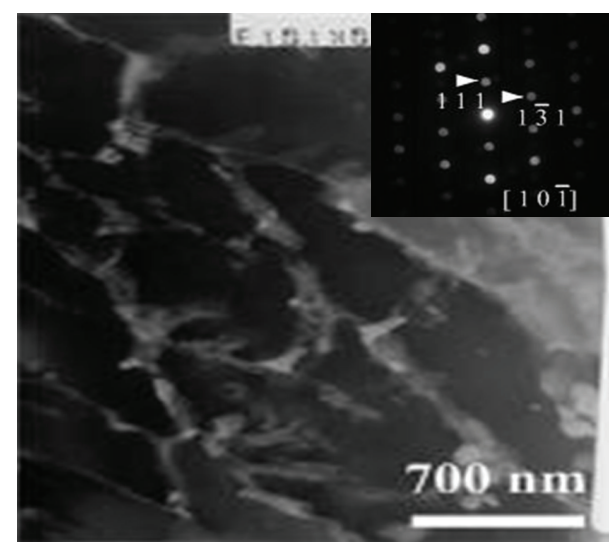

(c)

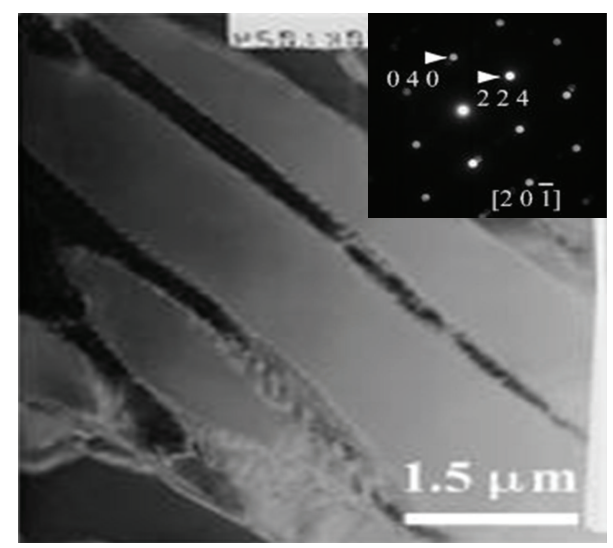

(e)

FIGURE 5: XRD pattern (a) and bright-field TEM images with corresponding SAED patterns (b-e) for location \#3. The crystalline phases in (b), (c), (d), and (e) are the cubic $\mathrm{AlZr}_{3}$, the orthorhombic $\mathrm{Al}_{3} \mathrm{Zr}_{2}$, the hexagonal $\mathrm{Al}_{3} \mathrm{Zr}_{5}$, and the orthorhombic $\mathrm{Ni}_{10} \mathrm{Zr}_{7}$, respectively. 


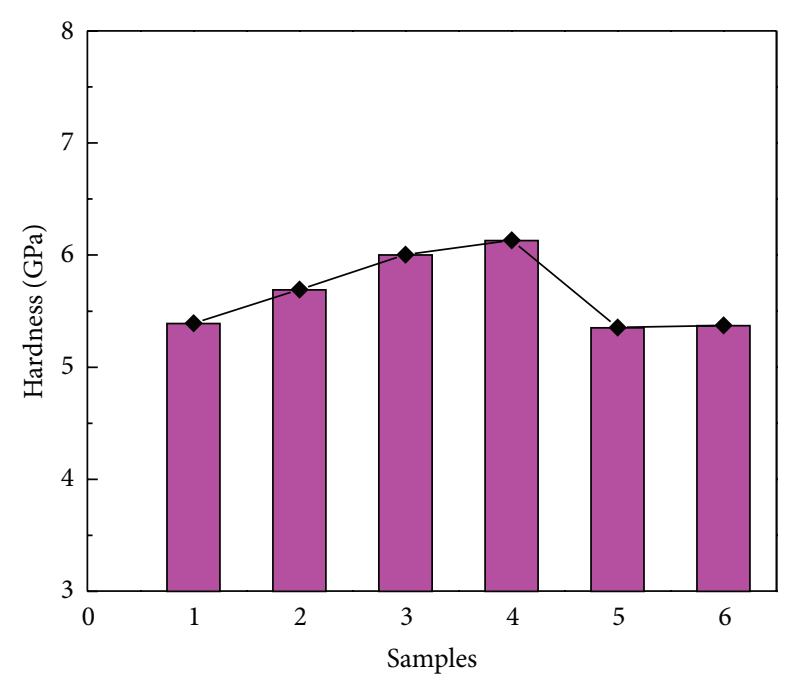

FIGURE 6: Vickers' hardness changes for different locations showing hardness marks for location \#1 \#6.

prohibit the nucleation and growth of the crystalline phase, crystallites then come into being.

Microstructure corresponding to the center part of the sample, location \#3, is shown in Figure 5. It clearly can be seen from the XRD pattern in Figure 5(a) there are many sharp peaks over the diffuse halo base, which indicate that with further decreasing the cooing rate, more and more in situ crystallization occurs. When examined by TEM, in location \#3, less amorphous structure can been seen in the bright field image but a complex in situ crystallized microstructure. There exist four crystalline phases in all: (1) cubic $\mathrm{AlZr}_{3}$ $(a=0.4372 \mathrm{~nm})$, with a snowflake-like morphology in the bright-field image as shown in Figure 5(b); (2) orthorhombic $\mathrm{Al}_{3} \mathrm{Zr}_{2}(a=0.9598 \mathrm{~nm}, b=1.39 \mathrm{~nm}$, and $c=0.5572 \mathrm{~nm})$, with fractal-like morphology as displayed in Figure 5(c); (3) hexagonal $\mathrm{Al}_{3} \mathrm{Zr}_{5}(a=0.8184 \mathrm{~nm}$ and $c=0.5704 \mathrm{~nm})$ with peritectic-like morphology as displayed in Figure 5(d); (4) orthorhombic $\mathrm{Ni}_{10} \mathrm{Zr}_{7}(a=0.921 \mathrm{~nm}, b=0.916 \mathrm{~nm}$, and $c=$ $1.239 \mathrm{~nm}$ ), with a strip morphology as shown in Figure 5(e).

What is surprising is that no hexagonal $\mathrm{Zr}_{5} \mathrm{Al}_{4}$ phase was found in the center of this rod sample. Therefore, it can be inferred that this hexagonal $\mathrm{Zr}_{5} \mathrm{Al}_{4}$ is a medium metastable phase which can only primarily crystallize during cooling the melt but disappear in the end. However, the emergence of the $\mathrm{Zr}_{5} \mathrm{Al}_{4}$ phase makes it easier for the succeeding crystalline phases to nucleate and grow. Associating with decreasing the cooling rate, it is difficult to suppress the occurrence of nucleation and growth of crystallites, which then makes the glass formation ability (GFA) deteriorate. Of course, all of these crystalline phases are competing phases with the metallic glass. As a result, in order to improve the GFA of the BMG, constraints between these competing phases must be improved by increasing the cooling rate, and combined with the proper compositions, the nucleation and growth of the crystalline phase will be more difficult.
3.2. Mechanical Property Evolution with Different Cooling Rates. The mechanical property discussed here concentrates on the microhardness which is very sensitive to the microstructure. Figure 6 shows the trend of microhardness evolution from edge to center tested in locations \#1 \#6. It can be seen that, from edge, medium to center, the Vickers' hardness gradually increases. Around the edge, the microhardness is relatively low, but in the center it owns the highest value. This difference is mainly due to the microstructure evolution resulting from the varying cooling rates [11]. As discussed in Section 3.1, the microstructure of the sample evolves with decreasing cooling rate as follows: full amorphous nature around the edge, amorphous matrix with dispersed hexagonal $\mathrm{Zr}_{5} \mathrm{Al}_{4}$ crystallites in the medium part, and amorphous matrix with crystalline phases of cubic $\mathrm{AlZr}_{3}$, orthorhombic $\mathrm{Al}_{3} \mathrm{Zr}_{2}$, hexagonal $\mathrm{Al}_{3} \mathrm{Zr}_{5}$, and orthorhombic $\mathrm{Ni}_{10} \mathrm{Zr}_{7}$ in the center part. Therefore, similar as reported in [13], the decreasing cooling rate leads to the precipitation of complex crystalline phases, which then results in the increase in the microhardness.

Figure 7 gives the SEM images for the Vickers' indentations in the Zr51 alloy (here, we only take into account the cases in locations \#1, \#2, and \#3 owing to the symmetrical location), where Figures 7(a), 7(b), and 7(c) correspond to the indentation morphologies in locations $\# 1, \# 2$, and \#3. It can be found that around the edge, there are multiple shear bands (pile-up) surrounding the indented region, which closely correlates with the plastic deformation ability of BMG [15]. As a result, the accumulation of so many shear bands indicates good plasticity during the hardness measurements. However, when moving into the center, the plasticity decreases as inferred by the disappearing shear bands detected in locations $\# 2$ and $\# 3$.

According to free volume models [16], generally, the free volumes in metallic glasses and their composites are very easy to generate during cooling. And the formation of shear bands around the indents closely relates to the free volume, which can be explained like this: when the indenter touches the surface of the sample, atoms surrounding the indents are punched into the free volume. On one hand, rearrangement of atomic structure occurs in these areas, and a certain irreversible deformation follows at the same time which is the plastic deformation happening before the initiation of shear bands. On the other hand, new free volume will form under the indentation, which then accumulates in those narrow areas. Because of the constraints from the geometry of the indenter, when the free volume is trapped here, sliding follows in advance of the fracture. With the succeeding interaction under the indentation, more and more localized areas form and then the sliding follows. It is this gradual deformation that leaves these multiple shear bands around the indented region [15].

Therefore, from the above analysis, it can be inferred that the more the free volume and viscous flow units in the material, the better the plasticity. Around the edge of the sample, real cooling rate is very high, which results in more free volume in the amorphous matrix. As a result, it owns a lot of plastic deformation and multiple shear bands after indentation as shown in Figure 7(a). However, 


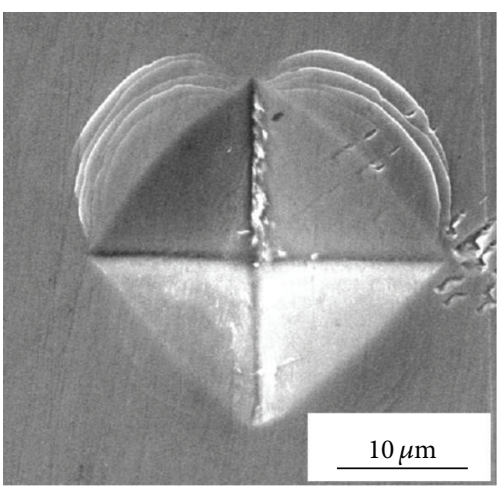

(a)

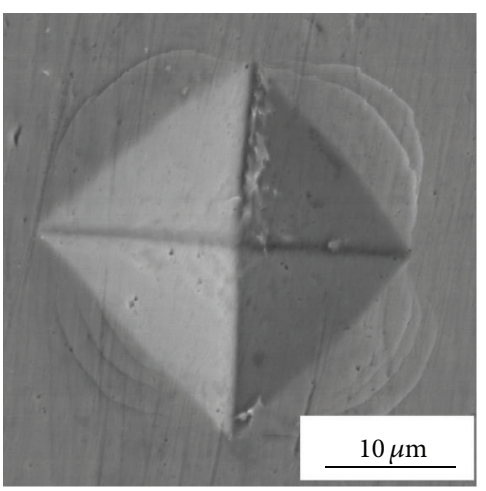

(b)

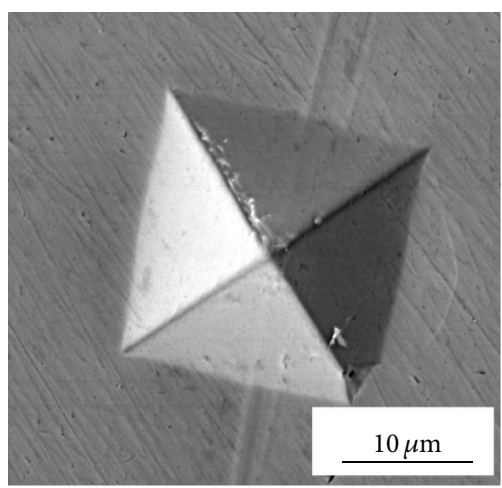

(c)

FIGURE 7: Morphologies of Vickers' indentation for different positions of Zr51 alloys under loading 500 g: (a) with multiple shear bands for outer location \#1, (b) with a small quantity of shear bands for middle location \#2 and (c) without shear bands for center location \#3.

in the medium part as indicated by location $\# 2$, diminished cooling rate allows the atoms to move more easily. It then makes the atomic packing density increase and brings into partial crystallization. All of these lead to a decrease in the free volume and then the plasticity. At the same time, due to the increased atomic packing density, the resistance to deformation also increases, which directly results in an increasing microhardness.

\section{Conclusion}

Throughout the $12 \mathrm{~mm}$ diameter $\mathrm{Zr}_{51} \mathrm{Al}_{9.96} \mathrm{Ni}_{14.34} \mathrm{Cu}_{24.9}$ (Zr51) alloy sample, there is a gradient of crystallization due to uneven cooling rate upon solidification. From the edge to center, microstructure evolves as almost amorphous matrix, amorphous matrix with dispersed hexagonal $\mathrm{Zr}_{5} \mathrm{Al}_{4}$ crystallites, and amorphous matrix with cubic $\mathrm{AlZr}_{3}$, orthorhombic $\mathrm{Al}_{3} \mathrm{Zr}_{2}$, hexagonal $\mathrm{Al}_{3} \mathrm{Zr}_{5}$, and orthorhombic $\mathrm{Ni}_{10} \mathrm{Zr}_{7}$ crystalline phases. Higher cooling rate results in lower hardness with more shear bands around the indents. The plasticity is ascribed to high fraction of free volume around the edge of the sample.

\section{Acknowledgments}

This work was supported by the Tianjin Research Program of Application Foundation and Advanced Technology no. 13JCQNJC02900 and the National Nature Science Foundation of China under Grant no. 11104201.

\section{References}

[1] W. L. Johnson, "Bulk glass-forming metallic alloys: science and technology," MRS Bulletin, vol. 24, no. 10, pp. 42-56, 1999.

[2] W. H. Wang, C. Dong, and C. H. Shek, "Bulk metallic glasses," Materials Science and Engineering Reports, vol. 44, no. 2-3, pp. 45-89, 2004.

[3] B. Zhang, M. X. Pan, D. Q. Zhao, and W. H. Wang, "'Soft' bulk metallic glasses based on cerium," Applied Physics Letters, vol. 85, no. 1, pp. 61-63, 2004.
[4] F. Guo, S. J. Poon, and G. J. Shiflet, "Metallic glass ingots based on yttrium," Applied Physics Letters, vol. 83, no. 13, pp. 2575$2577,2003$.

[5] W. H. Wang, "Roles of minor additions in formation and properties of bulk metallic glasses," Progress in Materials Science, vol. 52, no. 4, pp. 540-596, 2007.

[6] M. F. Ashby and A. L. Greer, "Metallic glasses as structural materials," Scripta Materialia, vol. 54, no. 3, pp. 321-326, 2006.

[7] C. A. Schuh, T. C. Hufnagel, and U. Ramamurty, "Mechanical behavior of amorphous alloys," Acta Materialia, vol. 55, no. 12, pp. 4067-4109, 2007.

[8] A. Inoue, "Stabilization of metallic supercooled liquid and bulk amorphous alloys," Acta Materialia, vol. 48, no. 1, pp. 279-306, 2000.

[9] X. Hu, S. C. Ng, Y. P. Feng, and Y. Li, "Cooling-rate dependence of the density of $\mathrm{Pd}_{40} \mathrm{Ni}_{10} \mathrm{Cu}_{30} \mathrm{P}_{20}$ bulk metallic glass," Physical Review B, vol. 64, no. 17, Article ID 172201, 4 pages, 2001.

[10] Y. Hu, K. C. Chan, L. Liu, and Y. Z. Yang, "Cooling rate induced variation in microstructure and magnetic structure of $\mathrm{Nd}_{60} \mathrm{Fe}_{30} \mathrm{Al}_{10}$ glass forming alloy," Materials Letters, vol. 61, no. 18, pp. 3909-3912, 2007.

[11] N. Chen, K. F. Yao, F. Ruan, and Y.-Q. Yang, "The influence of cooling rate on the hardness of Pd-Si binary glassy alloys," Materials Science and Engineering A, vol. 473, pp. 274-278, 2008.

[12] D. C. Hofmann, G. Duan, and W. L. Johnson, “TEM study of structural evolution in a copper mold cast $\mathrm{Cu}_{46} \mathrm{Zr}_{54}$ bulk metallic glass," Scripta Materialia, vol. 54, no. 6, pp. 1117-1122, 2006.

[13] M. Yan, J. Shen, J. F. Sun, and J. Zou, "Cooling rate dependent as-cast microstructure and mechanical properties of Zr-based metallic glasses," Journal of Materials Science, vol. 42, no. 12, pp. 4233-4239, 2007.

[14] K. Hildal, N. Sekido, and J. H. Perepezko, "Critical cooling rate for $\mathrm{Fe}_{48} \mathrm{Cr}_{15} \mathrm{Mo}_{14} \mathrm{Y}_{2} \mathrm{C}_{15} \mathrm{~B}_{6}$ bulk metallic glass formation," Intermetallics, vol. 14, no. 8-9, pp. 898-902, 2006.

[15] R. Vaidyanathen, M. Dao, and G. Ravichandran, "Study of mechanical deformation in bulk metallic glass through instrumented indentation," Acta Materialia, vol. 49, no. 16, pp. 37813789, 2001.

[16] P. S. Steif, F. Spaepen, and J. W. Hutchinson, "Strain localization in amorphous metals," Acta Metallurgica, vol. 30, no. 2, pp. 447$455,1982$. 

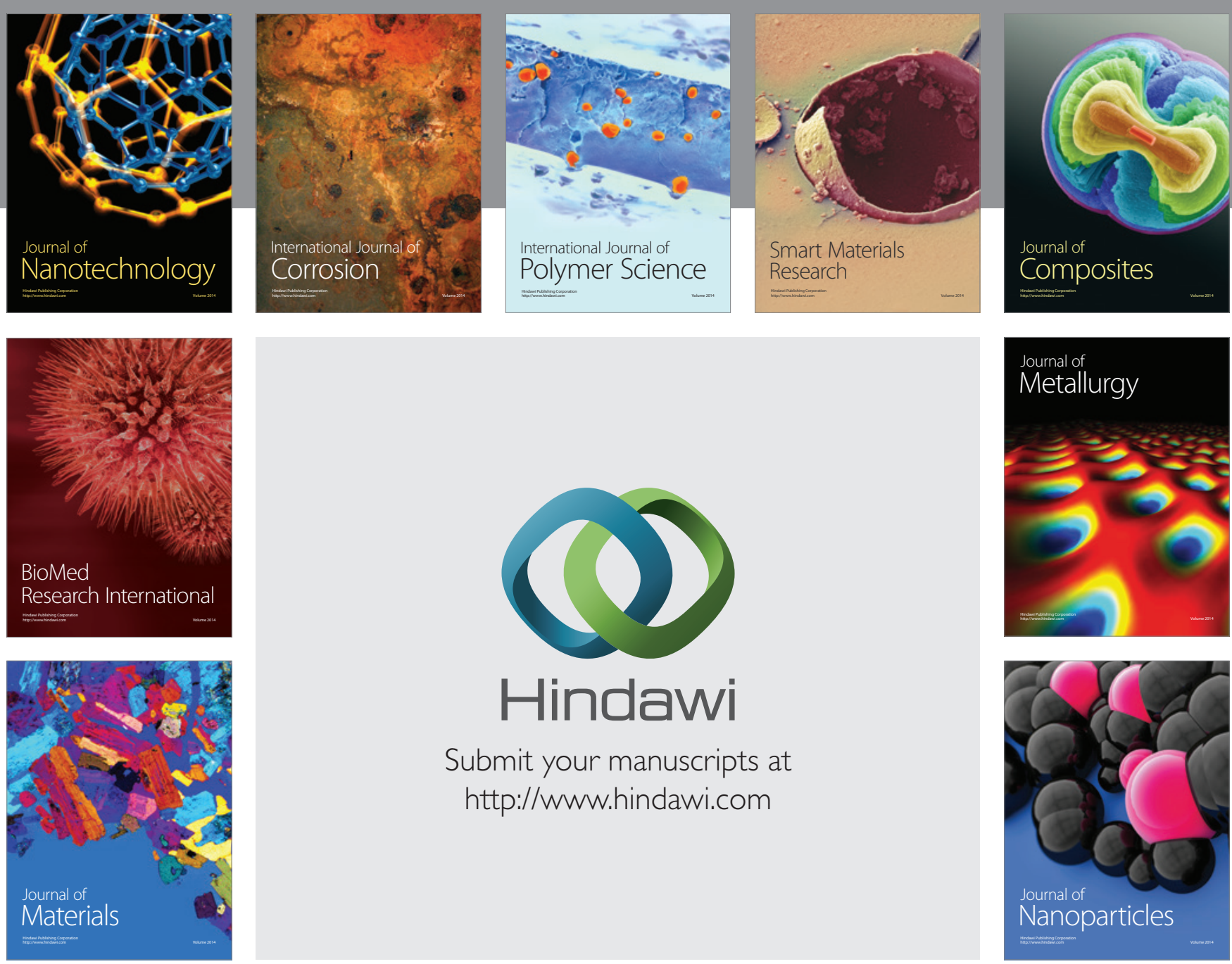

Submit your manuscripts at http://www.hindawi.com
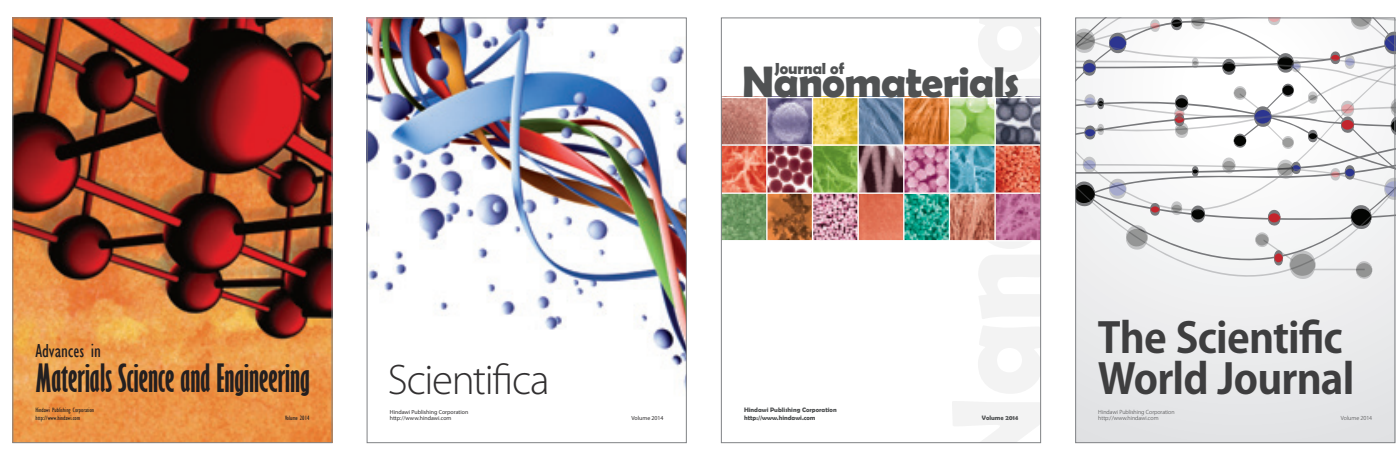

\section{The Scientific World Journal}
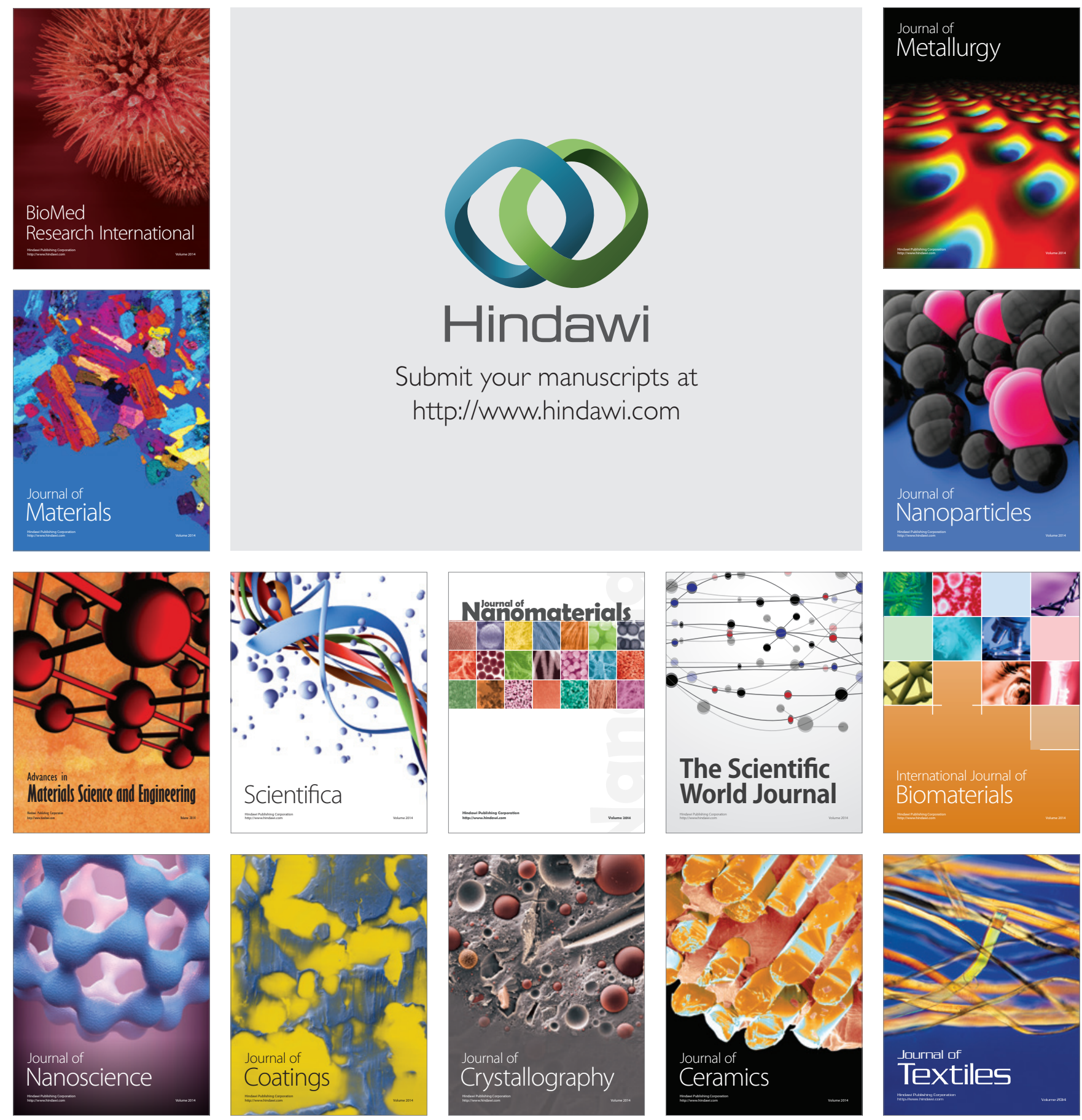